\title{
New ways with reptating polymers
}

A surprisingly simple model of polymer molecules moving in a network representing the constraints of others like themselves may be a pointer to a simpler theory of macromolecules in solution.

THE simplest thing to say about the thermodynamics of polymer solutions is that nothing is particularly simple. This, no doubt, is one of the reasons why attempts to calculate the properties of polymer systems seem ever to be striving for still simpler models. One of the neatest so far must be that of Michael Rubinstein, from the Eastman Kodak Company at Rochester, New York, who has produced a model for the movement of a polymer molecule that resembles in many ways the movement of the pieces on a backgammon board (Phys. Rev. Lett. 59,1946; 1987).

The essence of the problem is that polymer chains can become entangled with each other. In extreme cases, they can even become knotted together, simulating molecules twice as big, or even several times as big, as the starting material. But even when this has not happened, the movement of individual molecules will be constrained by the presence of neighbouring molecules.

What this means in practice is that not all the space a polymer molecule occupies is freely accessible to it. Some parts of the molecule may be relatively free to assume all possible configurations, but others will be constrained as if at bottlenecks there may be points, or regions of space represented perhaps by encircling loops of other molecules, through which they must pass.

The calculation of the movement of a polymer molecule, or even the mere enumeration of the configurations accessible to it, is a little like estimating the ease with which a long snake can thread its way through a series of bottlenecks along its path. But snakes usually know where they are heading, whereas the motion of polymer molecules in solution is strictly statistical. There is nothing to prevent the movement of polymer molecules being impeded by their own perverse tendency to bunch themselves together in ways that make their passage through bottlenecks that much more difficult.

Plainly all calculations of such a state of affairs must be simplifications. The ground rules are essentially those due to S.F.Edwards (now Sir Sam Edwards of the Cavendish Laboratory, Cambridge) in the 1960s: think of a single polymer molecule in a three-dimensional liquid as being confined within a tube of variable diameter, representing the external constraints of other molecules. Then set out to calculate the chance that the molecule will move as a whole along the tube in one direction or the other, allowing for the way in which, by chance, different lengths of polymer may be bunched together in successive sections of the tube. This phenomenon, called 'reptation', is what happens when real snakes move.

One of the standard simplifications of the problem of movement by reptation is to suppose that the space accessible to a molecule is defined by some kind of regular lattice. In two dimensions, the ground rules would have it that the backbone of a molecule should not be allowed to cross a lattice point. In three dimensions, the corresponding rule is that polymer tracks entering a cell through one face cannot cross any of the edges bounding that face.

The essence of Rubinstein's further simplification of the problem is his concept of 'reptons', or sections along the length of the molecule (which ideally should be of roughly equal length). Then it is possible to represent a configuration of the molecule as a statement of the number of reptons to be found in successive cells along the prescribed track of the molecule. Naturally, in such a description, there can be no gaps along the track, for that would mean that the backbone of the molecule is broken.

The movement of molecules is now stripped down to, and perhaps beyond, its bare essentials. Rubinstein's recipe is to calculate the statistical properties of these collections of bunched reptons in successive cells under the assumption that each of them is free to move by random walking in either direction subject only to two constraints, which are as follows: (1) the configuration can never correspond to a broken chain, with an empty cell separating two clusters, and (2) the chance that a repton will move into an empty contiguous cell is greater than that it will move into a contiguous occupied cell by the factor $(z-1)$, where $z$ is the number of faces of each cell, or its coordination number.

The statistical mechanics of this model are breathtakingly simple. With $N$ reptons per molecule, the calculation of the number of configurations in which the reptons are distributed over only $K$ contiguous cells (where $K \leqslant N$ ) is simply the number of ways in which $N-K$ objects can be distributed among $K$ sites. From this combinatorial way of putting things, it quickly emerges that the average value of $K$, the cluster size, is proportional to the total number of reptons $N$, which is what everybody expects.

The surprise is that this simple model also yields an estimate of the diffusion constant of the molecule. The first step is to calculate the chance that a jump of the chain from one repton distribution to another will succeed, which is a simple if tricky piece of probability calculation. Taking the diffusion constant as measured by the rate at which the centre of mass of the system moves along its tube, the simple result (in three dimensions) is that the diffusion constant is inversely proportional to the square of the molecular mass (which is the correct result).

This by itself is well-known. Rubinstein's special claim is that his simple model also yields a basis for calculating the relaxation time of a polymer molecule, which is in turn related to the experimental quantity of polymer solutions, the viscosity. The physics underlying this calculation is also childishly simple - to tackle the question of the rate at which a bunched-up polymer molecule will move when one of its ends is tugged (biasing the directions of likely random walks near the pulled end of the chain).

Rubinstein is exercised by this point after noting that there is a glaring discrepancy between previous predictions of the relationship between longest relaxation time of a polymer molecule and its molecular weight (a cube-law dependence) and empirical values (which yield a power-law dependence with an exponent more like 3.4).

As always in these circumstances, the argument concludes with numerical simulations. Rubinstein calculates from his simple model values of the relaxation time or viscosity of longish polymer molecules and concludes that there is no reason why the exponent of the power-law dependence on molecular weight should not be anywhere between 3.22 and 3.52, depending on the value of $z$. By itself, that proves nothing. The model may be too simple to be a good guide to reality. And who, in any case, can tell what may happen in the real world of polymer molecules, where $z$ may change from one cell along the backbone of the molecule to the next. Yet this is a long way to have come with such a stripped-down model of polymer solutions.

John Maddox 